\title{
Cerebral ischemia/reperfusion injury induces airway mucus hypersecretion in rats and activates IL-13-associated inflammatory mechanisms
}

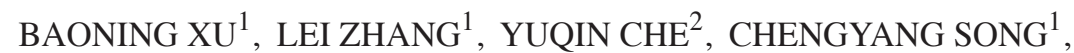 \\ WENJUN JIANG $^{1}$, JIANHUA FAN ${ }^{2}$ and DALI TIAN ${ }^{1}$ \\ Departments of ${ }^{1}$ Thoracic Surgery and ${ }^{2}$ Neurology, The Fourth Affiliated Hospital of China Medical University, \\ Shenyang, Liaoning 110032, P.R. China
}

Received March 5, 2016; Accepted March 13, 2017

DOI: $10.3892 / \mathrm{mmr} .2017 .7516$

\begin{abstract}
The majority of patients that suffer a stroke have excessive sputum, which accelerates the development of pulmonary complications. However, it is unclear whether cerebral ischemia and reperfusion (I/R) injury induces mucus hypersecretion, and the potential role of inflammation remains unknown. In the present study, the reversible middle cerebral artery occlusion model was applied in rats to induce cerebral $\mathrm{I} / \mathrm{R}$ injury. The rats were grouped according to the duration of reperfusion (6, 12, 24, 48 and 72 h). Neurological dysfunction was evaluated by Longa scoring and lung dry-to-wet weight (dw/ww) ratios were determined to reflect the degree of mucus secretion. Inflammatory factor interleukin-13 (IL-13) and tumor necrosis factor- $\alpha(\mathrm{TNF}-\alpha)$ levels in serum and bronchoalveolar lavage fluid (BALF) were determined by enzyme-linked immunosorbent assay. Pulmonary levels of mucin 5AC (MUC5AC) and key molecules involved in nuclear factor $-\kappa \mathrm{B}(\mathrm{NF}-\kappa \mathrm{B})$ signaling were determined by western blotting and immunohistochemistry. Rats with cerebral I/R had impaired neurological function, which was associated with the length of reperfusion time. In addition, the dw/ww lung ratio decreased and the pulmonary expression of MUC5AC increased with the increase in severity of neurological dysfunction, indicating that cerebral I/R may induce mucus hypersecretion in a reperfusion time-dependent manner. IL-13 and TNF- $\alpha$ levels in serum and BALF, as well as the nuclear translocation of NF- $\mathrm{BB}$ p 65 in pulmonary tissues, significantly increased following cerebral I/R, which suggests that the activation of IL-13 and NF- $\mathrm{B}$ inflammatory pathways may be involved. The present study concluded that cerebral I/R
\end{abstract}

Correspondence to: Dr Wenjun Jiang, Department of Thoracic Surgery, The Fourth Affiliated Hospital of China Medical University, 4th Chongshan East Road, Shenyang, Liaoning 110032, P.R. China

E-mail: wenjunjiang321@sina.com

Key words: cerebral ischemia, reperfusion, inflammation, mucus hypersecretion, interleukin-13 injury may induce airway mucus hypersecretion by activating IL-13 and NF- $\mathrm{BB}$ inflammatory pathways.

\section{Introduction}

Previous clinical studies have revealed that pulmonary complications, including acute respiratory distress syndrome (1), severe pneumonia (2) and respiratory failure, are important causes of mortality in patients with ischemic stroke, particularly in the elderly. Hilker et al (3) first reported the epidemiological and prognostic impact of the incidence of pneumonia for the treatment of acute stroke and demonstrated that the occurrence of stroke-associated pulmonary complications deteriorate the clinical outcomes in these patients. However, the mechanisms underlying the fact that patients with acute ischemic stroke are vulnerable to respiratory complications have not been clearly elucidated, despite their clinical significance for the early prevention of stroke-associated pulmonary syndrome and in improving clinical outcomes $(4,5)$.

Mucus hypersecretion has been recognized as one of the important features of airway remodeling and is involved in the pathogenesis of a number of pulmonary diseases, including chronic pulmonary obstructive diseases (6) and asthma (7). Although mucus secretion by goblet cells in the airway epithelium is thought to protect the respiratory system in physiological conditions, the pathological overproduction of mucus often leads to increased sputum production, airway narrowing due to sputum secretion and increased airway wall thickness $(8,9)$. The majority of patients with acute stroke have increased sputum retention in the airway, which is potentially induced by factors involving the overproduction of the mucus, saliva secretion and dysphagia secondary to the neurologic dysfunction (1). It is possible that the overproduction of airway mucus may be an early sign of airway dysfunction that occurs prior to onset and it may accelerate the onset of severe pulmonary disorders. However, it remains unclear whether cerebral ischemia induces airway mucus hypersecretion and the potential mechanisms underlying this pathophysiologic process have not been studied extensively.

A previous study indicated that the inflammatory response has an important role in the pathogenesis of mucus 
hypersecretion (10). T-helper 2 (Th2) cell-associated cytokines, particularly interleukin-13 (IL-13), are thought to be key regulators of mucus production in the airway (11). In addition, classical inflammatory signaling via the nuclear factor- $\kappa \mathrm{B}$ $(\mathrm{NF}-\kappa \mathrm{B})$ signaling pathway, has also been indicated to be involved in the regulation of mucus overproduction during pathological airway remodeling $(12,13)$. Increased levels of cytokines, such as IL-13 and other factors, trigger the activation of the NF- $\mathrm{NB}$ pathway in pulmonary tissues, which functions as a transcriptional factor with the nuclear translocation of its key component p65. This subsequently leads to cellular processes including the overproduction of mucus and airway remodeling. Notably, cerebral ischemia and reperfusion (I/R) injury, which frequently occurs in patients following stroke, has been associated with systematic activated inflammatory responses (14). Previous studies have indicated that patients with acute ischemic stroke have a higher degree of immunoinflammatory responses, which is reflected by increased serum levels of inflammatory markers (15) and alterations in the peripheral frequency of $\mathrm{CD} 4^{+}$cells (16). In addition, the degree of the immunoinflammatory response, which is characterized by higher peripheral white blood cell count at admission, has been associated with increased in-hospital mortality and cognitive impairment at discharge for patients with acute cerebrovascular syndromes (17). Anti-inflammation therapies, such as treatments targeting tumor necrotic factor- $\alpha$ (TNF- $\alpha$ ), have been proposed as a potential therapeutic strategy for patients with brain injuries from strokes and trauma (18). These findings indicate that activation of the inflammatory response serves a key role in the pathogenesis and progression of cerebral I/R injury. Therefore, we hypothesized that cerebral I/R injury may lead to airway mucus hypersecretion via the activation of systematic inflammatory responses, particularly those associated with the IL-13 and NF- $\mathrm{BB}$ signaling pathways.

Therefore, in the present study, the aim was to investigate the above hypothesis in a rat model of cerebral I/R by evaluating the influence of cerebral I/R injury on mucus secretion in the airway and the expression of its key component, mucin 5AC (MUC5AC). To further elucidate the potential mechanisms involved, the influence of cerebral I/R injury on systematic and pulmonary IL-13 and activation of the NF- $\kappa$ B pathway were also examined.

\section{Materials and methods}

Ethics statement. All animal experiments were performed in accordance with the National Institutes of Health Guide for the Care and Use of Laboratory Animals (19) and the Helsinki Convention on the Use and Care of Animals. The Ethics Committee of the Fourth Affiliated Hospital to China Medical University approved the experimental protocols prior to the commencement of the present study.

Animal models of cerebral I/R injury and evaluation of neurological dysfunction. Healthy male Sprague Dawley rats $(n=40$; age, 3 to 4 months; weight, 280 to $300 \mathrm{~g}$ ) were provided by the Animal Experimental Research Center of China Medical University. Rats were housed in the light for $14 \mathrm{~h}$ and the dark for $10 \mathrm{~h}$, at a temperature of $23 \pm 2^{\circ} \mathrm{C}$ and a humidity of $60-70 \%$, with food and water freely available. A total of 7 rats were randomly allocated to the sham group (control), while the remaining 33 rats were allocated to the cerebral I/R group. Following satisfactory anesthesia with $10 \%$ chloral hydrate (350 $\mathrm{mg} / \mathrm{kg}$; intraperitoneal injection), the middle cerebral artery occlusion (MCAO) method was used to establish the model of cerebral I/R injury in rats. Rats in the sham group were subjected to the same surgical procedures performed on the rats in the I/R group, however, their blood vessels were not occluded. The animals were not allowed to eat or drink $12 \mathrm{~h}$ prior to the operation. A rat model of focal cerebral ischemia was induced according to the modified Longa method, as previously described [Longa et al (20)].

Briefly, a $0.23-\mathrm{mm}$ diameter fish-thread was inserted into the right common carotid artery through a mini-pore, which was 3-mm away from the bifurcation. Then, the thread was inserted through the bifurcation and into the internal carotid artery until it had advanced $\sim 18$ to $20 \mathrm{~mm}$ to induce focal ischemia in the brain. Following MCAO for $2 \mathrm{~h}$, the operator carefully removed the suture to restore blood flow, sutured the skin and allowed the rat to wake up. Sham-operated animals underwent the same surgical procedure, except for arterial occlusion. The rectal temperatures of the rats from each group were monitored during the surgical process. Rats in the cerebral I/R group were further randomized into five subgroups according to the different reperfusion time $(6,12$, 24,48 and 72 h). Neurological function was evaluated immediately following reperfusion in the brains of rats in each group. Severity of neurological dysfunction was assessed based on the standards of Longa [Longa et al (20)]: Score 0, the rat had no symptoms of neurological deficit; score 1, the rat failed to fully to extend the left forepaw; score 2 , the rat circled to the left; score 3, the rat fell to the left; score 4, the rat did not walk spontaneously and had a depressed level of consciousness.

Collection of serum samples and bronchoalveolar lavage fluid $(B A L F)$. Following evaluation of neurological function, rats from each group were sacrificed with an overdose of sodium pentobarbital $(150 \mathrm{mg} / \mathrm{kg}$; Euthatal, Merial; Boehringer Ingelheim Corporation, Ridgefield, CT, USA), which was injected intraperitoneally. Serum was collected from the posterior vena cava using a heparinized syringe and plasma was separated by centrifugation $\left(1,000 \mathrm{x}\right.$ g for $20 \mathrm{~min}$ at $\left.4^{\circ} \mathrm{C}\right)$. The thoracic cavity was carefully opened and the trachea was exposed. BALF was collected by cannulating the upper part of the trachea and performing lavage twice using $1 \mathrm{ml}$ and $0.8 \mathrm{ml}$ of phosphate-buffered saline (PBS), respectively; 85 to $90 \%$ of the total input volume was recovered. BALF samples were kept on ice during collection then centrifuged at $400 \mathrm{x} g$ for $5 \mathrm{~min}$ at $4^{\circ} \mathrm{C}$.

Determination of dry-to-wet lung weight ratio. The wet weight (ww) was recorded following collection then $100 \mathrm{mg}$ of lung tissue was dried in an oven at $60^{\circ} \mathrm{C}$ for $48 \mathrm{~h}$ to reach a constant weight termed the dry weight $(\mathrm{dw})$. The $\mathrm{dw} / \mathrm{ww}$ ratio was calculated, which was used as an indicator of pulmonary mucus production.

Measurement of IL-13, TNF- $\alpha$ and MUC5AC protein levels in serum and BALF. Serum and BALF supernatants were collected as described above and stored at $-70^{\circ} \mathrm{C}$ for use in cytokine 
assays. IL-13 (cat. no. SEM03021A; Qiagen, Inc., Valencia, CA, USA), TNF- $\alpha$ (cat. no. SEM03113A; Qiagen, Inc.) and MUC5AC (cat. no. fk2954Y; R\&D Systems, Inc., Minneapolis, MN, USA) levels were measured using enzyme-linked immunosorbent assay (ELISA) kits, according to the manufacturer's protocol. All assays were performed in duplicate, and the mean values were used for statistical analysis.

Immunohistochemical analysis. The right lower lung obtained from each rat was fixed in $10 \%$ formalin for 10 min, embedded in paraffin, cut into $5-\mu \mathrm{m}$ sections and stained with $0.2 \%$ hematoxylin at room temperature for $10 \mathrm{~min}$ and $0.5 \%$ eosin at room temperature for $20 \mathrm{sec}$. MUC5AC, aquaporin-5

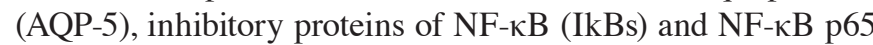
in lung tissues were detected by immunohistochemistry. Tissue sections were deparaffinized, treated with $\mathrm{H}_{2} \mathrm{O}_{2}$ and blocked with 5\% normal rabbit serum in PBS at room temperature for $1 \mathrm{~h}$. Following washing with PBS, tissue sections were incubated with rabbit anti-MUC5AC (cat. no. sc-20118; 1:1,000), anti-AQP-5 (cat. no. sc-28628; 1:500), anti-IkBs (cat. no. sc-847; 1:500) and anti-NF- $\kappa$ B p65 (cat. no. sc-372; 1:500) antibodies (Santa Cruz Biotechnology, Inc., Dallas, TX, USA) overnight at $4^{\circ} \mathrm{C}$. Following rinsing with PBS, the sections were incubated with biotinylated anti-rabbit IgG (cat. no. sc-2040; Santa Cruz Biotechnology, Inc.) for at $4^{\circ} \mathrm{C} 2 \mathrm{~h}$. Specific binding was detected with an avidin-biotin-horseradish peroxidase complex and diaminobenzidine kit (Vector Laboratories, Inc., Burlingame, CA, USA). Slides were then counterstained with hematoxylin, dehydrated using graded alcohol and xylenes, and mounted on coverslips. The sections were examined using an LSM 5 PASCAL confocal microscope (Carl Zeiss AG, Oberkochen, Germany). Negative control staining was performed using normal bovine serum instead of a primary antibody. Two blind investigators analyzed the sections using the Image-Pro Plus software version 6.0 (Media Cybernetics, Inc., Rockville, MD, USA). The positive areas were evaluated in $\geq 12$ randomly selected tissue sections from each of the groups.

Western blot analysis. Total proteins in lung tissue samples were ground and homogenized using a protein lysis solution (Nanjing KeyGen BioTech Co., Ltd., Nanjing, China) for $\geq 12$ randomly selected tissue samples from each group studied. Briefly, the left upper lung tissues were frozen in liquid nitrogen and homogenized in PBS with a protease inhibitor cocktail (Roche Applied Science, Pleasanton, CA, USA) using a tissue grinder. Homogenates were centrifuged at $21,920 \mathrm{x}$ g for $15 \mathrm{~min}$ at $4^{\circ} \mathrm{C}$. Nuclear and cytoplasmic proteins were separated according to the protocol described in the Nuclear and Cytoplasmic Extraction kit (Thermo Fisher Scientific, Inc., Waltham, MA, USA). Supernatants were collected and assayed for total protein using a bicinchoninic kit (Nanjing KeyGen BioTech Co., Ltd.), according to the manufacturer's protocol. Equal amounts of protein $(125 \mu \mathrm{g})$ were resolved on $10 \%$ Tris-glycine-sodium dodecyl sulfate (SDS) polyacrylamide gels, and protein bands were blotted onto nitrocellulose membranes. With the aid of the pre-stained markers, the gel for MUC5A, AQP-5, and $\beta$-actin was cut at $\sim 70 \mathrm{kDa}$. The larger molecular weight portion was used for staining of MUC5A, and the smaller molecular weight portion was used for staining of AQP-5. $\beta$-actin was subsequently stained after stripping of the membrane. Similarly, the gel for $\mathrm{cNF}-\kappa \mathrm{B}, \mathrm{IkBs}$ and $\beta$-actin was cut at $\sim 50 \mathrm{kDa}$. The larger molecular weight portion was used for staining of $\mathrm{cNF}-\kappa \mathrm{B}$, and the smaller molecular weight portion was used for staining of IkBs. Again, $\beta$-actin was subsequently stained after stripping of the membrane. The nuclear gel was stained for $n N F-\kappa B$ and subsequently with Lamin A after stripping of the membrane. Following blocking with $5 \%$ dried milk in Tris-buffered saline containing $0.1 \%$ Tween-20 for $1 \mathrm{~h}$ at room temperature, membranes were incubated for $24 \mathrm{~h}$ at $4^{\circ} \mathrm{C}$ with one of the following antibodies to detect protein levels: Anti-MUC5AC (cat. no. sc-20118; 1:500), anti-AQP-5 (cat. no. sc-28628; 1:1,000), anti-IkBs (cat. no. sc-847; 1:500), anti-NF-кB p65 (cat. no. sc-372; 1:1,000), anti- $\beta$-actin (cat. no. sc-47778; 1:5,000) and anti-Lamin A (cat. no. sc-293162; 1:500), purchased from Santa Cruz Biotechnology, Inc. Membranes were incubated for $1 \mathrm{~h}$ at room temperature with horseradish peroxidase-conjugated rabbit anti-mouse (cat. no. sc358914; 1:10,000; Santa Cruz Biotechnology, Inc.) or a donkey anti-rabbit secondary antibody (cat. no. sc2315; 1:10,000; Santa Cruz Biotechnology, Inc.). The internal controls including $\beta$-actin and Lamin A were detected after stripping of the membranes, which was performed using stripping buffer (cat. no. P0025N; Beyotime Institute of Biotechnology, Haimen, China). In brief, after rinsing the membrane with distilled water for $5 \mathrm{~min}$, they were incubated with stripping buffer for 10 mins at room temperature and then washed with PBS 3 times. Peroxidase labeling was detected using the enhanced chemiluminescence western blotting detection system (Amersham Pharmacia Biotech; GE Healthcare Life Sciences, Chalfont, UK) and analyzed by densitometry using Image-Pro Plus software version 6.0 (Media Cybernetics, Inc.). Experiments were repeated three times. Optical density values were normalized to that of $\beta$-actin or Lamin A.

Statistical analysis. Statistical analyses were performed using SPSS 11.0 (SPSS, Inc., Chicago, IL, USA). Results are presented as the mean \pm standard deviation. Differences were analyzed for significance by one-way or two-way analysis of variance followed by the least significant difference post hoc test. $\mathrm{P}<0.05$ was considered to indicate a statistically significant difference.

\section{Results}

During the surgical procedure for the induction of cerebral I/R injury, 1 rat in the sham group and 3 rats in the I/R group did not survive. In total, 6 rats were included in the sham group and 30 rats were included in the I/R group for the present study. In the I/R group, rats were further divided into subgroups with 6 rats in each subgroup according to the different reperfusion times $(6,12,24,48$ and $72 \mathrm{~h})$. All of the rats that survived the surgical procedures underwent subsequent neurological evaluation, and pathological and molecular experiments.

Focal cerebral I/R injury-induced neurological dysfunction. Using a conventional revisable MCAO model revealed that focal ischemia, and the subsequent reperfusion to the brain, was associated with various degrees of neurological dysfunction in rats in the I/R group. Although rats in the sham operated group did not exhibit any obvious symptoms of neurological disorder, rats that underwent cerebral ischemia and subsequent 


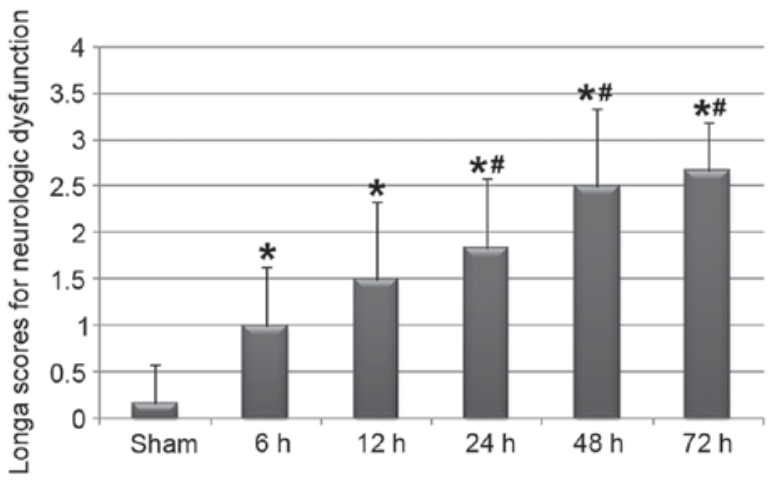

Figure 1. Severity of neurological dysfunction was evaluated by the Longa scoring system [Longa et al (20)] in rats from the sham group and the cerebral I/R group following 6,12, 24, 48 and $72 \mathrm{~h}$ reperfusion time (n=6/group). Data are presented as the mean \pm standard deviation. " $\mathrm{P}<0.05$ vs. sham group; ${ }^{\#} \mathrm{P}<0.05$ vs. cerebral I/R with $6 \mathrm{~h}$ of reperfusion. I/R, ischemia and reperfusion.

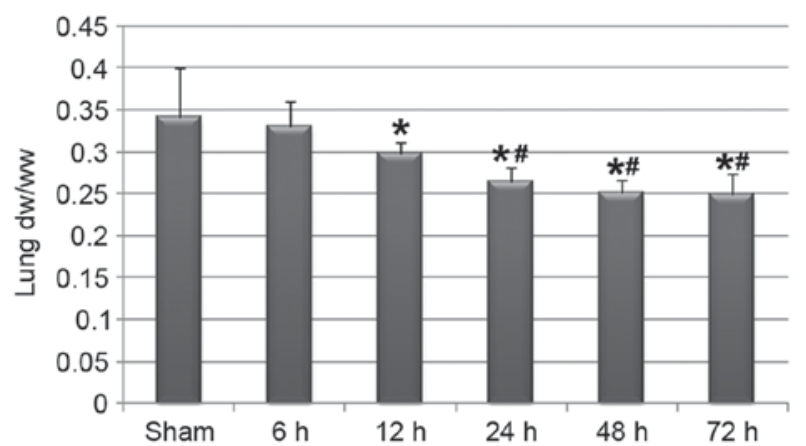

Figure 2. Lung dw/ww ratios in rats from the sham group and the cerebral I/R group following 6, 12, 24, 48 and $72 \mathrm{~h}$ of reperfusion ( $\mathrm{n}=6$ /group). Data are presented as the mean \pm standard deviation. ${ }^{~} \mathrm{P}<0.05$ vs. sham group; ${ }^{"} \mathrm{P}<0.05$ vs. cerebral I/R with 6 h of reperfusion. dw/ww, lung dry-to-wet weight ratio; $\mathrm{I} / \mathrm{R}$, ischemia and reperfusion.

reperfusion for $\geq 6 \mathrm{~h}$ presented with various degrees of neurological disorder, as presented in Fig. 1. In addition, the severity of neurological dysfunction may be dependent on the reperfusion time of the brain.

Cerebral I/R injury was associated with airway mucus hypersecretion. The dw/ww ratio of the lung is thought to be reflective of the amount of mucus production in the airway. In the present study, cerebral I/R injury and the neurological dysfunctions were associated with a significant decrease in dw/ww ratio in the lung (Fig. 2), indicating that focal cerebral ischemia and the subsequent reperfusion may induce airway mucus hypersecretion. In addition, the reduction in the $\mathrm{dw} / \mathrm{ww}$ ratio may be associated with the reperfusion time of the brain.

Cerebral I/R injury-activated inflammatory factors in serum and $B A L F$. As the activation of the inflammatory response has been recognized as an important mechanism underlying the pathogenesis of cerebral I/R injury $(14,21)$, and the inflammatory factors IL-13 and TNF- $\alpha$ have been proposed to be key regulators of pulmonary mucus production in a number of pulmonary diseases (10), the present study continued to evaluate whether cerebral I/R injury was associated with the
A

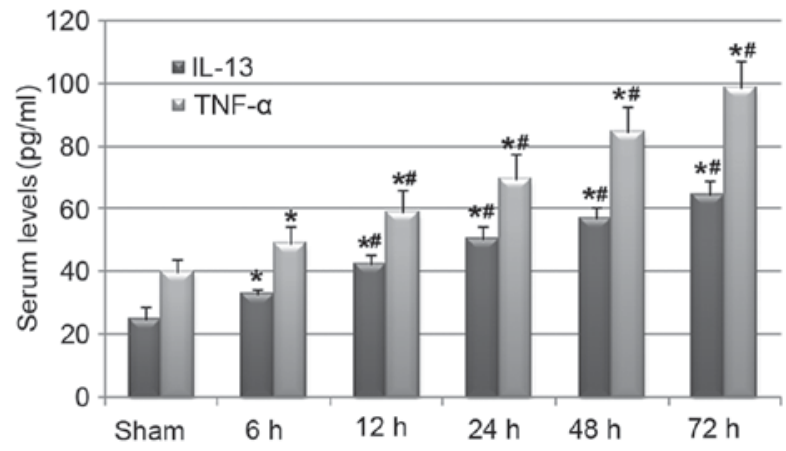

B

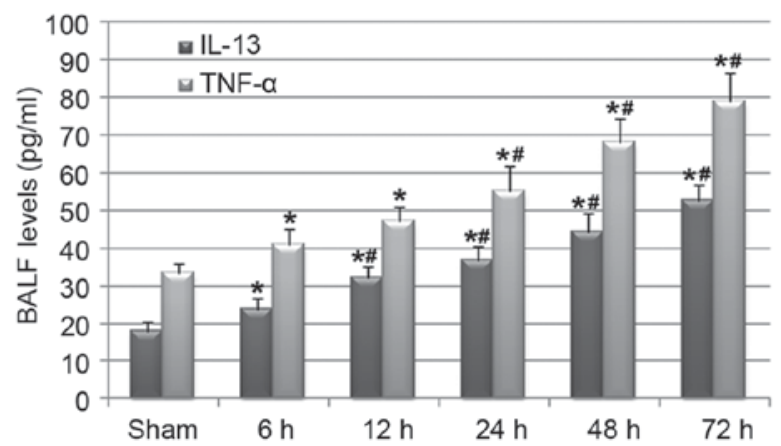

Figure 3. IL-13 and TNF- $\alpha$ levels in the (A) serum and (B) BALF of rats in the sham group and the cerebral I/R group following 6, 12, 24, 48 and $72 \mathrm{~h}$ of reperfusion ( $n=6 /$ group). Data are presented as the mean \pm standard deviation. ${ }^{*} \mathrm{P}<0.05$ vs. sham group; ${ }^{\#} \mathrm{P}<0.05$ vs. cerebral I/R with $6 \mathrm{~h}$ of reperfusion. IL-13, interleukin-13; TNF- $\alpha$, tumor necrosis factor- $\alpha$; I/R, ischemia and reperfusion; BALF, bronchoalveolar lavage fluid.

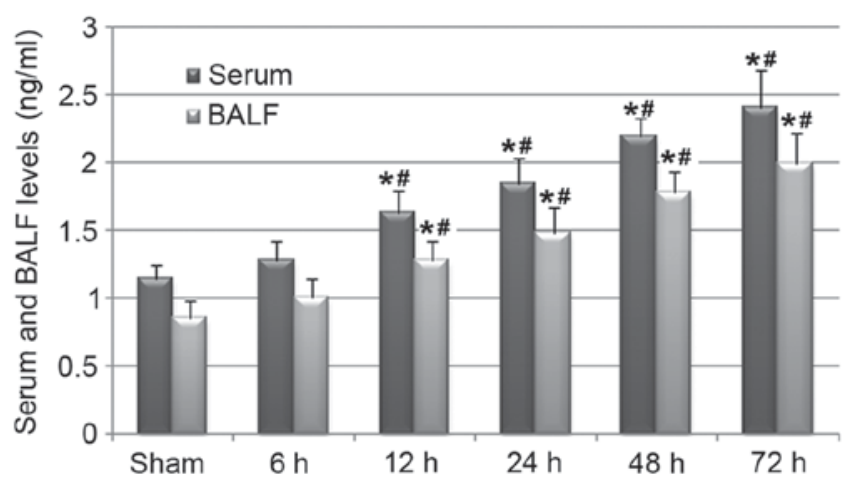

Figure 4. MUC5AC levels in the serum and BALF of rats in the sham group and the cerebral I/R group following $6,12,24,48$ and $72 \mathrm{~h}$ of reperfusion ( $\mathrm{n}=6$ /group). Data are presented as the mean \pm standard deviation. ${ }^{*} \mathrm{P}<0.05$ vs. sham group; ${ }^{~} \mathrm{P}<0.05$ vs. cerebral I/R group following $6 \mathrm{~h}$ of reperfusion. MUC5AC, mucin 5AC; BALF, bronchoalveolar lavage fluid; I/R, ischemia and reperfusion.

activation of these factors in the serum and BALF of rats in the I/R group. Notably, cerebral I/R injury upregulated IL-13 and TNF- $\alpha$ serum levels (Fig. 3A) and also increased BALF levels of these cytokines (Fig. 3B) in a reperfusion time-dependent manner. These results indicate that the activation of the systematic inflammatory response, reflected by the increase in IL-13 and TNF- $\alpha$ levels in serum and BALF, may mediate the pathological process of cerebral I/R injury-induced airway mucus hypersecretion. 
A
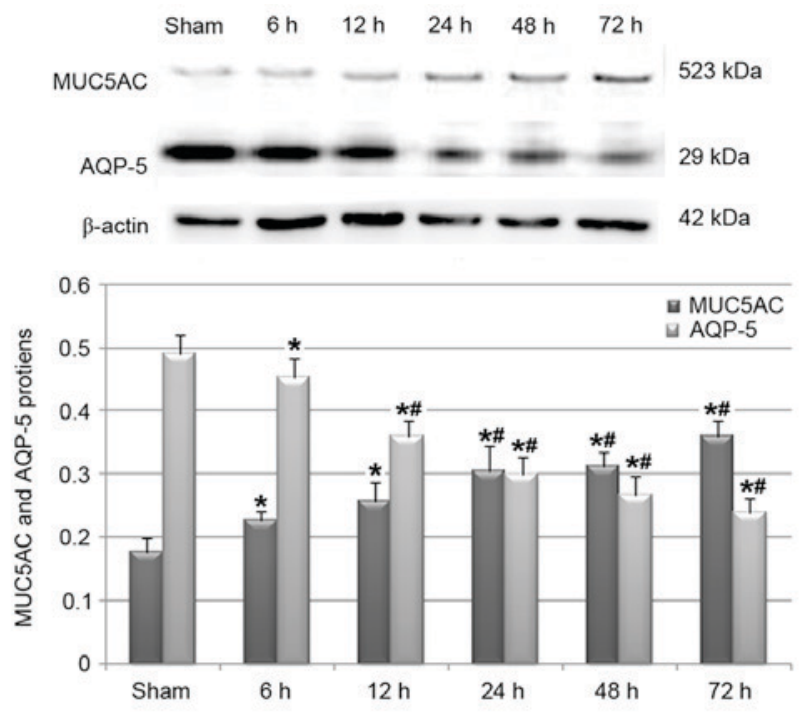

C

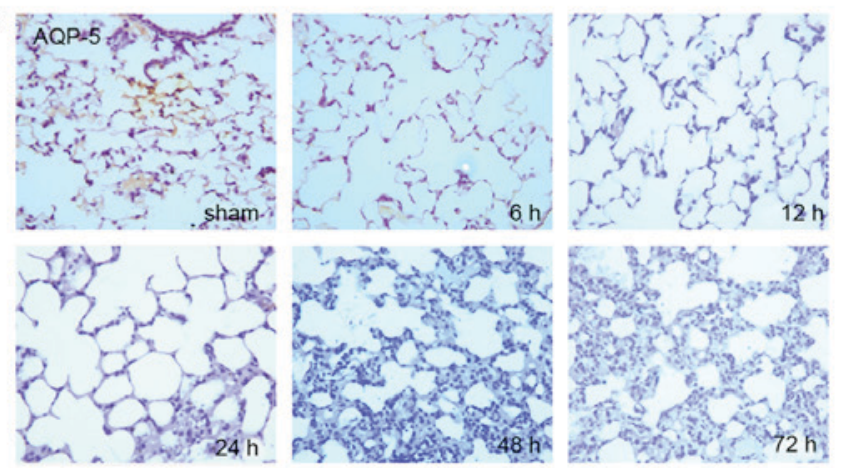

B

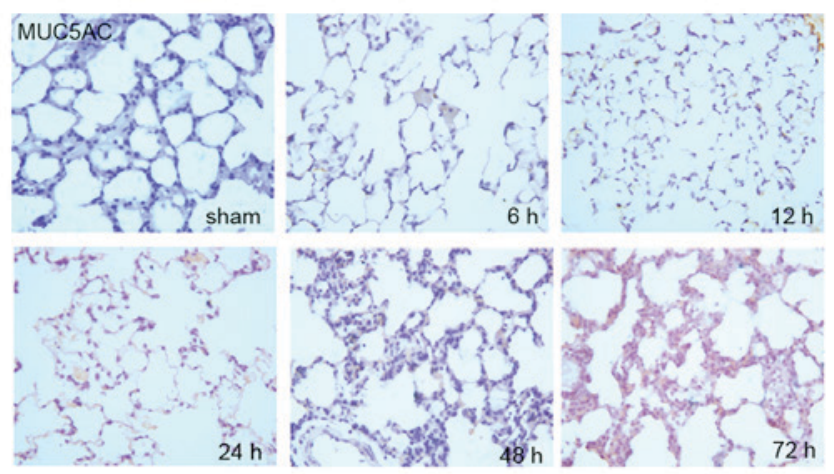

D

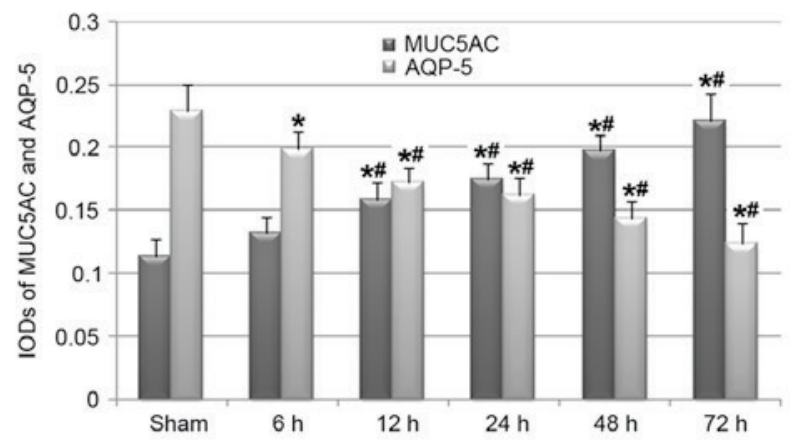

Figure 5. Effects of cerebral I/R injury on the pulmonary expression of MUC5AC and AQP-5. Western blot and immunohistochemical analyses were performed in rats in the sham group and the cerebral $\mathrm{I} / \mathrm{R}$ group following $6,12,24,48$ and $72 \mathrm{~h}$ of reperfusion (n=6/group). (A) Pulmonary expression of MUC5AC and AQP-5 as detected by western blot analysis. The upper panel shows representative images of the western blotting study and the lower panel displays the quantitative results of pulmonary MUC5AC and AQP-5 expression. Optical density values of MUC5AC and AQP-5 were normalized to that of $\beta$-actin. (B) Representative images (magnification, $\mathrm{x} 400$ ) of the immunohistochemical analysis of pulmonary MUC5AC in rat lung tissues in each group. (C) Representative images (magnification, x400) of the immunohistochemical analysis of pulmonary AQP-5 in rat lung tissues in each group. Data are presented as the mean \pm standard deviation. (D) Quantitative results of pulmonary MUC5AC and AQP-5 expression presented as IOD values detected by immunohistochemical analysis. Each experiment was independently performed for 3 times. " $\mathrm{P}<0.05$ vs. sham group; $\mathrm{P}<0.05$ vs. cerebral $\mathrm{I} / \mathrm{R}$ group following $6 \mathrm{~h}$ of reperfusion. I/R, ischemia and reperfusion; MUC5AC, mucin 5AC; AQP-5, aquaporin 5; IOD, integrated optical density.

Cerebral I/R injury-induced pulmonary overexpression of MUC5AC. MUC5AC is the main component of mucus $(7,22)$. Therefore, the present study evaluated whether cerebral I/R injury was associated with the systematic and pulmonary overexpression of MUC5AC. ELISA analysis results revealed that rats in the 12-72 $\mathrm{h} \mathrm{I/R}$ groups had significantly increased levels of MUC5AC in serum and BALF (Fig. 4). These results were further confirmed by the western blot analysis of the MUC5AC protein in lung tissues, which revealed that cerebral I/R injury stimulated the pulmonary production of MUC5AC protein (Fig. 5A), potentially in a reperfusion time-dependent manner. A subsequent immunohistochemical study also confirmed that the pulmonary protein of MUC5AC was induced in rats in the I/R group (Fig. 5B). In addition, western blot analysis (Fig. 5A) and the immunohistochemical study (Fig. 5C and D) revealed that AQP-5, which contributes to the volume of liquid secreted from the airways (23), significantly decreased in the I/R group rats when compared with the rats in the sham group.

Cerebral I/R injury-activated $N F-\kappa B$ inflammatory signaling pathway in lungs. As NF- $\mathrm{\kappa B}$ inflammatory signaling has been implicated in the regulation of airway mucus hypersecretion $(12,13)$, we hypothesized that cerebral I/R injury may lead to the overproduction of mucus via activation of the pulmonary NF- $\kappa$ B pathway. Western blot analysis revealed that cerebral $\mathrm{I} / \mathrm{R}$ injury was associated with decreased protein levels of IkBs and cytoplasmic NF- $\mathrm{kB}$ p65, as well as increased levels of nuclear NF-кB p65 (Fig. 6A). These results indicate that there may be increased translocation of NF- $\mathrm{kB}$ p 65 to the nucleus and the subsequent activation of the NF- $\kappa \mathrm{B}$ inflammatory signaling pathway. In addition, changes in the expression of these proteins may be dependent on reperfusion time. These results were further confirmed by immunohistochemical analyses for IkBs and cytoplasmic NF- $\kappa \mathrm{B}$ p65 (Fig. 6B-D). Therefore, the results suggest that cerebral I/R injury may contribute to increased mucus production in the airway by activating the NF- $\mathrm{KB}$ inflammatory signaling pathway.

\section{Discussion}

In the present study, the rat model of cerebral I/R injury revealed that brain I/R injury-associated neurological dysfunction may be 
A

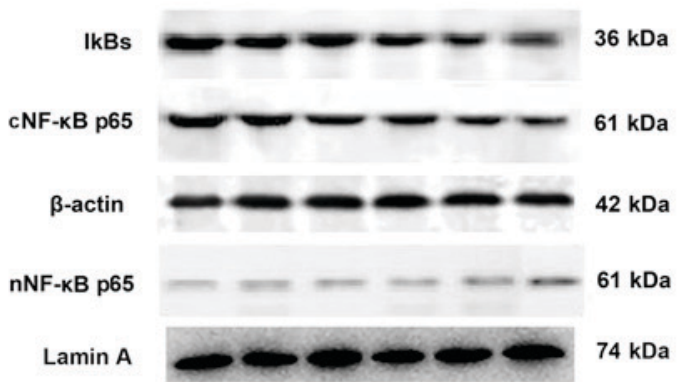

$\mathrm{C}$

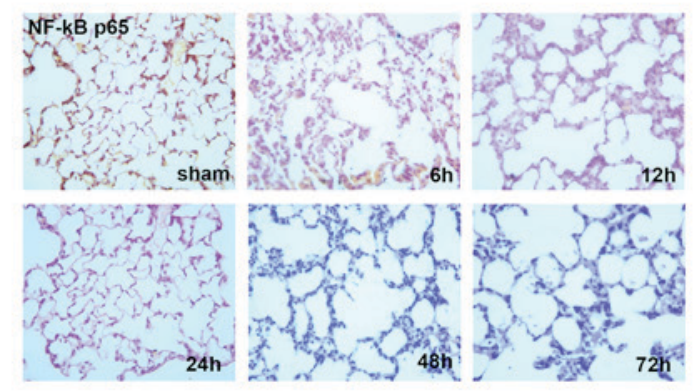

B
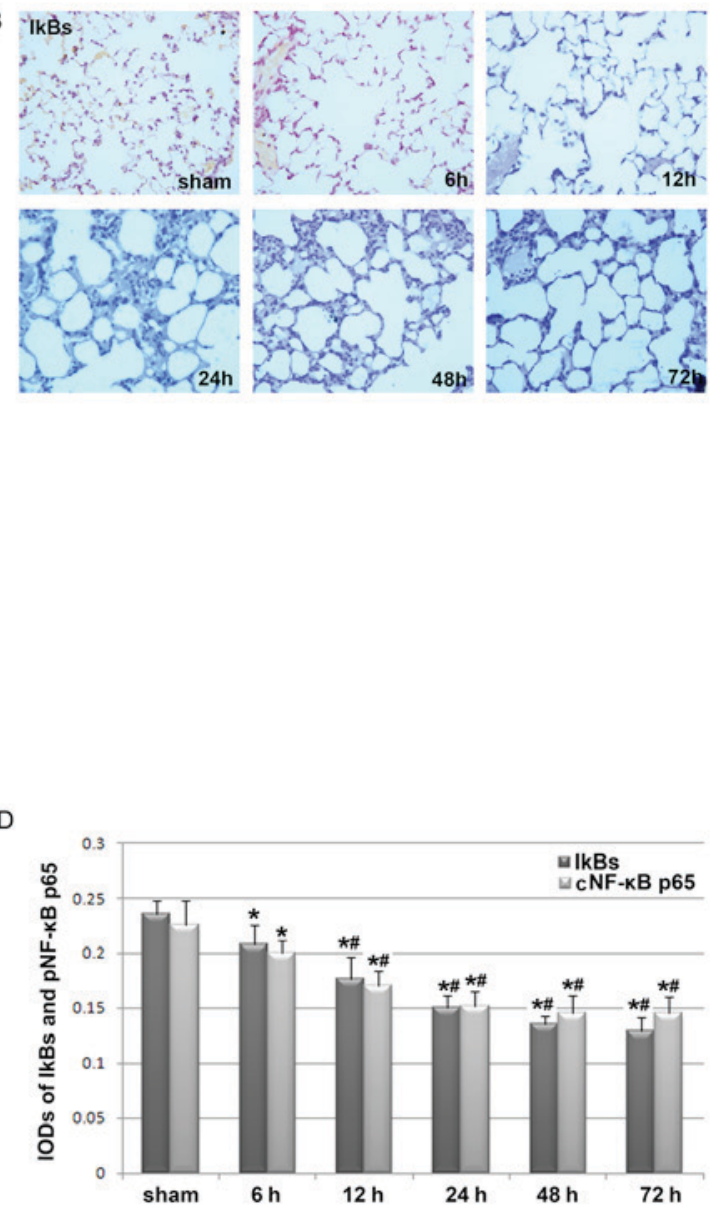

Figure 6. Effects of cerebral I/R injury on the pulmonary expression of IkBs and NF- $\mathrm{kB}$ p65. Western blot and immunohistochemical analyses were performed in rats in the sham group and the cerebral I/R group following 6, 12, 24, 48 and $72 \mathrm{~h}$ of reperfusion (n=6/group). (A) Pulmonary expression of IkBs, and cytoplasmic and nuclear NF-kB p65 detected by western blot analysis. The upper panel shows the representative images of the western blotting study, while the lower panel displays the quantitative results of pulmonary IkBs, and cytoplasmic and nuclear NF-kB p65 expression. Optical density values of IkBs, and cytoplasmic NF-kB p65 were normalized to that of $\beta$-actin, while the optical density of nuclear NF-kB p65 was normalized to that of Lamin A. (B) Representative images (magnification, $\mathrm{x} 400$ ) for the immunohistochemical analysis of pulmonary IkBs in rat lung tissues in each group. (C) Representative images (magnification, $\mathrm{x} 400$ ) for the immunohistochemical analysis of pulmonary cytoplasmic NF- $\mathrm{KB}$ p65 in rat lung tissues in each group. (D) Quantitative results of the pulmonary expression of IkBs and NF- $\mathrm{KB}$ p65 presented as IOD values detected by immunohistochemical analysis. Each experiment was independently performed for 3 times. Data are presented as the mean \pm standard deviation. ${ }^{*} \mathrm{P}<0.05$ vs. sham group; ${ }^{\#} \mathrm{P}<0.05$ vs. cerebral I/R group following

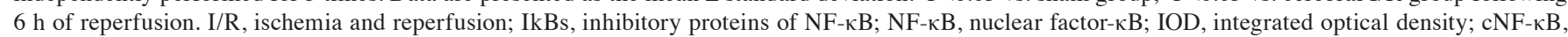

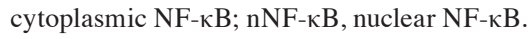

associated with mucus overproduction in the airway. In addition, the results of the subsequent mechanistic evaluations indicate that the upregulation of the Th2-associated inflammatory cytokine IL-13 and activation of the NF- $\kappa \mathrm{B}$ signaling pathway may be involved in the potential association between cerebral I/R injury and airway mucus hypersecretion. To the best of our knowledge, the present study is the first to elucidate the influence and potential mechanisms of brain I/R injury on airway mucus hypersecretion, which is of clinical significance. This highlights the fact that inflammation has an important role in the pathogenesis of brain I/R injury-induced mucus hypersecretion, and thus targeting inflammation may be a potential strategy for the early prevention of mucus hypersecretion and the associated pulmonary disorders for patients with ischemic stroke.

Previous clinical observations have determined that patients with neurological disorders are more vulnerable to respiratory complications $(1,2)$. In addition, comorbidities of pulmonary diseases often deteriorate the prognosis of patients with stroke (24). However, the potential pathophysiological association between brain I/R injury and pulmonary dysfunction has not been extensively investigated. In light of the fact that airway mucus hypersecretion has been suggested as an initial process in the pathogenesis of a number of pulmonary disorders (7), the present study investigated whether cerebral I/R-related neurological dysfunction was associated with the overproduction of mucus in the airway. Decreased dw/ww ratio of the lung and enhanced expression of MUC5AC in pulmonary tissues were observed in rats with I/R-associated neurological dysfunction in a reperfusion time-dependent manner. Further studies are required to determine the pathophysiological role of mucus hypersecretion in animal models and patients with neurological disorders, and to determine whether strategies targeting mucus hypersecretion could improve prognosis in patients with early-stage cerebral I/R injury. 
The activation of inflammation has been indicated to be important in the pathogenesis of cerebral I/R injury $(14,25)$ and mucus hypersecretion-associated airway remodeling (26,27). Therefore, the present study subsequently investigated whether the activation of systematic and pulmonary inflammatory pathways was responsible for the potential link between cerebral I/R injury-associated dysfunction and airway mucus hypersecretion. Th2-associated inflammatory factor IL-13 was induced in rat serum and BALF. This is of particular interest as previous studies have demonstrated that IL-13 induces a number of features of allergic lung disease including airway hyperresponsiveness, goblet cell metaplasia and mucus hypersecretion, which all contribute to airway obstruction $(28,29)$. An early in vivo study demonstrated that CD4 Th cells stimulate mucus only through a common IL-13-mediated pathway (11). Lin et al (30) demonstrated that the transmembrane protein 16A may be involved in the process of IL-13-mediated mucus hypersecretion. Notably, an animal study performed by Ma et al (31) revealed that suppression of IL-13 with a vaccine inhibits chronic airway inflammation and the development of several key components of airway remodeling including mucus hypersecretion. In addition, this intervention was more effective during the earlier stages of chronic inflammation (31). This is of clinical significance as targeting the inhibition of IL-13 may be effective for the prophylaxis of dysfunction in patients with cerebral I/R injury-associated neurological dysfunction. Further studies are required to confirm this hypothesis.

The present study also revealed that the NF- $\mathrm{B}$ signaling pathway was activated in the pulmonary tissues of rats with cerebral I/R injury-associated airway mucus overproduction. This was to be expected as previous studies have indicated that the activation of the NF- $\kappa \mathrm{B}$ inflammatory signaling pathway serves an important role in the pathogenesis of mucus hypersecretion $(32,33)$. The present study revealed that the level of IkBs decreased and the translocation of NF- $\mathrm{BB}$ p65 from the cytoplasm to the nucleus increased in the pulmonary tissues of rats with cerebral I/R-associated mucus hypersecretion, indicating that activation of the $\mathrm{NF}-\kappa \mathrm{B}$ signaling pathway may be involved. In fact, a previous study has indicated that activation of the $\mathrm{NF}-\kappa \mathrm{B}$ signaling pathway during the regulation of mucus overproduction may be the downstream event following the induced systematic expression of IL-13 (30). Further studies are required to determine whether the upregulation of systematic IL-13 and activation of the pulmonary $\mathrm{NF}-\kappa \mathrm{B}$ pathway was causative in the pathogenesis of cerebral $\mathrm{I} / \mathrm{R}$ injury-associated airway mucus hypersecretion. In addition, it is important to further investigate effective strategies that target the $\mathrm{NF}-\kappa \mathrm{B}$ signaling pathway for the prevention of pulmonary disorders in patients with brain I/R injury.

The current study has limitations that should be noted when interpreting the results. Firstly, dw/ww ratios of the lungs were used as an index of the extent of mucus hypersecretion. However, this marker may not be directly associated with mucus hypersecretion, and may be affected by other pulmonary processes that occur following a stroke including neurogenic pulmonary edema or inflammation. Other direct parameters or studies, such as periodic acid-Schiff staining of the pulmonary tissues should be performed in future studies. Secondly, the present study only focused on the changes and the potential role of IL-13. The potential involvement and function of the other Th2-associated inflammatory factors should be evaluated in the future. Finally, the sample size of the experimental animals in each group was relatively small. Therefore, further investigations should be performed with a larger sample size to confirm our results.

In conclusion, cerebral I/R injury may induce airway mucus hypersecretion via the activation of the IL-13 and $\mathrm{NF}-\kappa \mathrm{B}$ inflammatory signaling pathways. The present study revealed that inflammatory-related mucus hypersecretion may be responsible for the association between cerebral I/R-related neurological dysfunction and the susceptibility of patients who have suffered a stroke for respiratory disorders. Further studies are required to determine whether targeting inflammation during the above pathological process is effective for the early prevention of cerebral I/R-associated pulmonary complications.

\section{Acknowledgements}

The present study was funded by the Scientific and Technologic Program of Liaoning Province (grant no. 2013408001).

\section{References}

1. Zhao JN, Liu Y and Li HC: Aspiration-related acute respiratory distress syndrome in acute stroke patient. PLoS One 10: e0118682, 2015.

2. Maeshima S, Osawa A, Hayashi T and Tanahashi N: Elderly age, bilateral lesions, and severe neurological deficit are correlated with stroke-associated pneumonia. J Stroke Cerebrovasc Dis 23: 484-489, 2014.

3. Hilker R, Poetter C, Findeisen N, Sobesky J, Jacobs A, Neveling $M$ and Heiss WD: Nosocomial pneumonia after acute stroke: Implications for neurological intensive care medicine. Stroke 34: 975-981, 2003.

4. Hannawi Y, Hannawi B, Rao CP, Suarez JI and Bershad EM: Stroke-associated pneumonia: Major advances and obstacles. Cerebrovasc Dis 35: 430-443, 2013.

5. Smith CJ, Kishore AK, Vail A, Chamorro A, Garau J, Hopkins SJ, Di Napoli M, Kalra L, Langhorne P, Montaner J, et al: Diagnosis of stroke-associated pneumonia: Recommendations from the pneumonia in stroke consensus group. Stroke 46: 2335-2340, 2015.

6. Martin C, Frija-Masson J and Burgel PR: Targeting mucus hypersecretion: New therapeutic opportunities for COPD? Drugs 74: 1073-1089, 2014.

7. Bergeron $\mathrm{C}$, Tulic $\mathrm{MK}$ and Hamid Q: Airway remodelling in asthma: From benchside to clinical practice. Can Respir J 17: e85-93, 2010.

8. Aikawa T, Shimura S, Sasaki H, Ebina M and Takishima T: Marked goblet cell hyperplasia with mucus accumulation in the airways of patients who died of severe acute asthma attack. Chest 101: 916-921, 1992.

9. Jenkins HA, Cool C, Szefler SJ, Covar R, Brugman S, Gelfand EW and Spahn JD: Histopathology of severe childhood asthma: A case series. Chest 124: 32-41, 2003.

10. Turner J and Jones CE: Regulation of mucin expression in respiratory diseases. Biochem Soc Trans 37: 877-881, 2009.

11. Whittaker L, Niu N, Temann UA, Stoddard A, Flavell RA, Ray A, Homer RJ and Cohn L: Interleukin-13 mediates a fundamental pathway for airway epithelial mucus induced by CD4 T cells and interleukin-9. Am J Respir Cell Mol Biol 27: 593-602, 2002.

12. Chen M, Lv Z, Zhang W, Huang L, Lin X, Shi J, Zhang W, Liang R and Jiang S: Triptolide suppresses airway goblet cell hyperplasia and Muc5ac expression via NF- $\mathrm{B}$ in a murine model of asthma. Mol Immunol 64: 99-105, 2015.

13. Kang JH, Hwang SM and Chung IY: S100A8, S100A9 and S100A12 activate airway epithelial cells to produce MUC5AC via extracellular signal-regulated kinase and nuclear factor- $\kappa \mathrm{B}$ pathways. Immunology 144: 79-90, 2015. 
14. Ahmad M, Dar NJ, Bhat ZS, Hussain A, Shah A, Liu H and Graham SH: Inflammation in ischemic stroke: Mechanisms, consequences and possible drug targets. CNS Neurol Disord Drug Targets 13: 1378-1396, 2014.

15. Tuttolomondo A, Pecoraro R, Di Raimondo D, Di Sciacca R, Canino B, Arnao V, Buttà C, Della Corte V, Maida C, Licata G and Pinto A: Immune-inflammatory markers and arterial stiffness indexes in subjects with acute ischemic stroke with and without metabolic syndrome. Diabetol Metab Syndr 6: 28, 2014.

16. Tuttolomondo A, Pecoraro R, Casuccio A, Di Raimondo D, Buttà C, Clemente G, Della Corte V, Guggino G, Arnao V, Maida C, et al: Peripheral frequency of $\mathrm{CD} 4{ }^{+} \mathrm{CD} 28$ - cells in acute ischemic stroke: Relationship with stroke subtype and severity markers. Medicine (Baltimore) 94: e813, 2015.

17. Tuttolomondo A,Pedone C,Pinto A,DiRaimondoD,FernandezP,Di Sciacca R and Licata G; Gruppo Italiano di Farmacoepidemiologia dell'Anziano (GIFA) researchers: Predictors of outcome in acute ischemic cerebrovascular syndromes: The GIFA study. Int J Cardiol 125: 391-396,2008.

18. Tuttolomondo A, Pecoraro R and Pinto A: Studies of selective TNF inhibitors in the treatment of brain injury from stroke and trauma: A review of the evidence to date. Drug Des Devel Ther 8 : 2221-2238, 2014

19. Committee for the Update of the Guide for the Care and Use of Laboratory Animals: Guide for the care and use of laboratory animals. 8th Edition. https://grants.nih.gov/grants/olaw/ Guide-for-the-Care-and-Use-of-Laboratory-Animals.pdf.

20. Longa EZ, Weinstein PR, Carlson S and Cummins R: Reversible middle cerebral artery occlusion without craniectomy in rats. Stroke 20: 84-91, 1989.

21. Haqqani AS, Nesic M, Preston E, Baumann E, Kelly J and Stanimirovic D: Characterization of vascular protein expression patterns in cerebral ischemia/reperfusion using laser capture microdissection and ICAT-nanoLC-MS/MS. FASEB J 19: 1809-1821, 2005.

22. Alagha K, Palot A, Sofalvi T, Pahus L, Gouitaa M, Tummino C, Martinez S, Charpin D, Bourdin A and Chanez P: Long-acting muscarinic receptor antagonists for the treatment of chronic airway diseases. Ther Adv Chronic Dis 5: 85-98, 2014.
23. Shen Y, Wang Y, Chen Z, Wang D, Wang X, Jin M and Bai C: Role of aquaporin 5 in antigen-induced airway inflammation and mucous hyperproduction in mice. J Cell Mol Med 15: 1355-1363, 2011.

24. Llinas RH: Ischemic stroke and ICU care. Semin Neurol 28: 645-656, 2008

25. Tuttolomondo A, Di Sciacca R, Di Raimondo D, Renda C, Pinto A and Licata G: Inflammation as a therapeutic target in acute ischemic stroke treatment. Curr Top Med Chem 9: 1240-1260, 2009.

26. Angelis N, Porpodis K, Zarogoulidis P, Spyratos D, Kioumis I, Papaiwannou A, Pitsiou G, Tsakiridis K, Mpakas A, Arikas S, et al: Airway inflammation in chronic obstructive pulmonary disease. J Thorac Dis 6 (Suppl 1): S167-S172, 2014.

27. McGovern AE and Mazzone SB: Neural regulation of inflammation in the airways and lungs. Auton Neurosci 182: 95-101, 2014

28. Mitchell J, Dimov V and Townley RG: IL-13 and the IL-13 receptor as therapeutic targets for asthma and allergic disease. Curr Opin Investig Drugs 11: 527-534, 2010.

29. Rayees S, Malik F, Bukhari SI and Singh G: Linking GATA-3 and interleukin-13: Implications in asthma. Inflamm Res 63 255-265, 2014.

30. Lin J, Jiang Y, Li L, Liu Y, Tang H and Jiang D: TMEM16A mediates the hypersecretion of mucus induced by Interleukin-13. Exp Cell Res 334: 260-269, 2015

31. Ma Y, Halayko AJ, Basu S, Guan Q, Weiss CR, Ma AG, HayGlass KT, Becker AB, Warrington RJ and Peng Z: Sustained suppression of IL-13 by a vaccine attenuates airway inflammation and remodeling in mice. Am J Respir Cell Mol Biol 48: 540-549, 2013.

32. Janssen-Heininger YM, Poynter ME, Aesif SW, Pantano C, Ather JL, Reynaert NL, Ckless K, Anathy V, van der Velden J, Irvin CG and van der Vliet A: Nuclear factor kappaB, airway epithelium, and asthma: Avenues for redox control. Proc Am Thorac Soc 6: 249-255, 2009.

33. MortazE, MasjediMR,Allameh A and Adcock IM: Inflammasome signaling in pathogenesis of lung diseases. Curr Pharm Des 18: 2320-2328, 2012. 